\title{
Framework of Silicon Photonics
}

\author{
Piyush Kurkure \\ B.E., Student, Department of Computer Engineering, Fr. Conceicao Rodrigues Institute of Technology (Mumbai \\ University), Navi Mumbai, India
}

\begin{abstract}
Earlier, Scientists used to perform various research on discovering an alternative for copper wires which are used for data transfer between the microchips but now we have found that replacement by using silicon as an optical medium. We simply replace the original electronic circuits with photonic circuits. The future of IC industry depends upon the speed and bandwidth requirements, but in metallic interconnections we have constraints on speed as well as bandwidth. This is a key enabling factor of bringing photonics to technology areas where the costs of implementation using other methods is expensive. Silicon photonics provides us with low-cost approach for overcoming the bottleneck of the high data transmission rate. Silicon photonics is attention-seeking technology that is revolutionizing in several application areas like computing, data centers, sensing. The main objective behind using silicon photonics is that CMOS-like fabrication ability it provides resulting in high-volume production that too in low cost. It is clear that silicon photonics has a dazzling future. This paper provides an overview of the progress and the growth of each component in silicon photonics, including modulators, detectors, and lasers compared to last few years.
\end{abstract}

Keywords: Silicon, semiconductor fabrication, waveguides, avalanche photodiodes, Mach-Zehnder (MZ).

\section{INTRODUCTION}

Silicon Photonics is generally a combination of two of the most important inventions of the 20th century i.e. the silicon integrated circuit and the semiconductor laser. Silicon photonics is the study and application of photonic systems which uses silicon as an optical medium in which data is transferred among computer chips by optical rays (laser light), which can carry much larger data in very less time than electrical conductors can.

As world is revolutionizing day by day, the increase in bandwidth requirement is also perceived and realized on large scale but the parasitic effects in current metallic interconnection have gradually become a major obstacle for further improvements, since electric signal attenuation and power dissipation rise dramatically with higher data rate. So, to overcome this bottleneck of the high data transmission rate, one possible solution could be manipulating optical interconnections, in which the information signals are carried by photons instead. Compared with electrons, photons have zero rest mass and zero charge, which means that they can travel at velocity of light without the interference with electromagnetic field, so optical systems can theoretically achieve signal transmission with much higher data rate and better stability than electrical system.

Intel fellow Mario Paniccia once said that: "If you had the capability of transferring data at 50Gps between two devices using Silicon photonics, you are talking about transferring an HD movie in less than a second." Further, if there is advancement in Silicon photonics it is observed that data transfer at 1Tbps is attainable in a cost-effective way.

Why only Silicon is preferred among all elements?

Silicon ( $\mathrm{Si}$ ) has been the mainstay of the electronics industry for more than 40 years. Si has an apparent window from the wavelength ranging from $1100 \mathrm{~nm}$ to $7000 \mathrm{~nm}$ approximately, which is far from being limited to the near-infrared (IR) communication band of $1300-1550 \mathrm{~nm}$. The silicon is usually patterned with sub-micrometre precision, into micro photonic components. Some excellent optical properties like, large thermal conductivity and optical damage threshold, also appear in Si. Furthermore, today's mature complementary metal-oxide semiconductor (CMOS) techniques could also allow low-cost, large-scale manufacturing for Si photonic devices. (Fig. 1) shows the variation of intensity and absorbance w.r.t. wavelengths associated in $\mathrm{nm}$, which describes its optical properties.

Silicon photonic devices can be used through our existing semiconductor fabrication techniques, and because silicon is already used as basic surface for most integrated circuits, it is possible to design hybrid devices in which the optical and electronic components are integrated with a single microchip, enabling it for faster data transfer over longer distances as estimated with conventional electronics, while using the efficiencies of Intel's high-volume silicon manufacturing.

Consequently, silicon photonics is being mainly researched by many electronics manufacturers like IBM and Intel, including some academic research groups. 

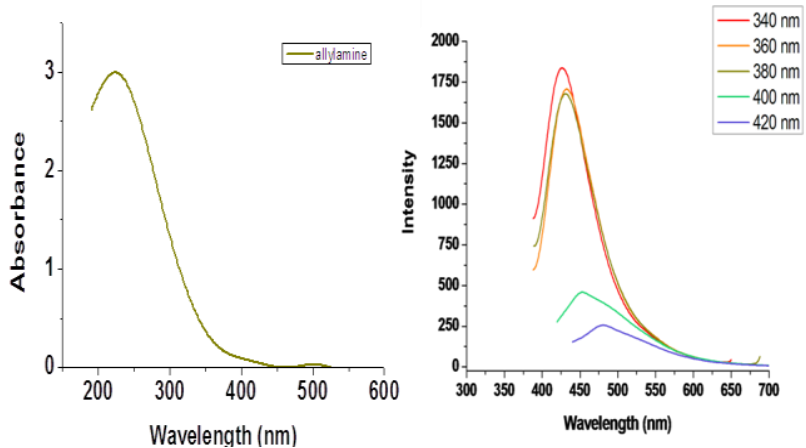

Fig. 1. Variation of intensity and absorbance w.r.t. wavelengths

This paper gives the basic idea of structures of these photonic systems that are accountable for Si based optic-electro combinations.

\section{PROPERTIES}

\section{A. Two photon absorption}

The performance of system is controlled by two-photon absorption, a process in which light is absorbed inside silicon with an intensity that depends on the square of the incoming light's intensity. It frequently heats the silicon and changes its refractive index generating free charge carriers (e.g., electrons), which further changes the refractive index of the silicon as well. The oscillations and frequency spectrum shows very interesting behaviour by discovering the interactions between heat, light and free charge carriers in disks. There is one major drawback of this absorption that it generates unwanted heat and wastes light. It can be overcome by switching to longer wavelengths or by using slot waveguides.

\section{B. Free charge carriers}

The free charge carriers inside silicon can absorb photons as well as change its refractive index. This is mostly found in high intensity interactions and situation when carrier concentration is saturated by TPA (generally in long durations). Scientist proposed many methods for removing these interactions among which one is the implantation of helium with silicon to enhance carrier recombination. The more advanced method to remove is to integrate the waveguide with intrinsic region of a PIN diode, which is reverse biased for making the carriers are attracted away from the waveguide core.

\section{The Raman effect}

Raman amplification in Silicon-on-Insulator waveguides is considered as a potential approach in developing active silicon photonic circuits. In this effect, a photon is exchanged with another photon with a slightly different energy, corresponding to an excitation or a relaxation of the material. The band size of Silicon's Raman transition is much narrower thus making it problematic for broadband phenomena such as Raman amplification, but is therefore beneficial for narrowband devices such as Raman lasers.

D. The Brillouin effect

Silicon waveguides is also supported by acoustic phonon excitations. Acoustic phonons are coherent movements of atoms of the lattice out of their equilibrium positions. Their interaction with light is known as Brillouin scattering. The frequencies these acoustic phonons is dependent on the geometry of the silicon waveguides. Their interactions with light make narrowband optical amplifiers.

\section{PASSIVE DEVICES}

For proper working of all integrated optical devices the basic requirement is low propagation losses in waveguides, couplers, Mach-Zehnder Interferometer and the cavities. In general, both size and propagation loss must be considered while evaluating the performance of a specific kind of optical device. There are actually two types of losses which are intrinsic loss which is the main loss for doping based waveguides like present in carrier absorption and another is extrinsic loss which comes into play due to change in field density at silicon surface and roughness at the interface. Surface roughness often lead to scattering losses. Consequently, silicon waveguides are characterized by losses in the range of $0.1-3 \mathrm{~dB} / \mathrm{cm}$. Thermal oxidation is performed to reduce the roughness of $\mathrm{Si} / \mathrm{SiO} 2$ interface on the waveguide sidewall with increasing temperature and oxidation time. A smooth sidewall without deformation of waveguide's crosssectional area is obtained when temperature reaches above $1000^{\circ} \mathrm{C}$. 
By doping III/V ions into the silica, the refractive index of the material will change around $0.1 \%$ to $0.75 \%$, resulting in change in refractive index between cladding and core. The refractive index contrast can be adjusted flexibly by changing doping density. The low refractive index means a weak confined structure for silica waveguides, which ultimately results in thickening of cladding layer (about $50 \mu \mathrm{m}$ ) and wide spacing between waveguides. A strong confinement is formed within the waveguides of silicon-on-insulator (SOI) i.e. $\mathrm{Si} / \mathrm{SiO} 2$ platforms, this occurs due to large refractive index contrast between $\mathrm{Si}(n=3.45)$ and $\mathrm{SiO} 2(n=1.45)$. This decreases the size of waveguides drastically to approximately $0.1 \mu \mathrm{m} 2$ on the same scale as typical dimensions encountered in CMOS VLSI.

Most recently, a novel type of converter based on suspended structure was reported by Fang et al. It consists of a suspended $\mathrm{SiO} 2$ waveguide in which $\mathrm{SiO} 2$ beams used to provide structural support and $\mathrm{Si}$ nanotapers were overlapped with each other present at the centre of the waveguide. The optical signal will first be launched into the suspended $\mathrm{SiO} 2$ waveguide and then be coupled with silicon nanotapers. The crucial advantage of this type is that it is almost wavelength independent and have a large alignment tolerance i.e. about $1.7 \mu \mathrm{m}$ for $1 \mathrm{~dB}$ excessive loss, thus overcoming the shortcoming of couplers in both taper type and grating type.

The optical filters are one of the important applications of passive devices to be used for wavelength multiplexing and demultiplexing. Arrayed waveguide grating, the workhorse of WDM communication, was demonstrated using SOI waveguides as early as 1997. More recently, microring and microdisk structures have been extensively explored for the same applications. For general applications, filters with wide free spectra range (which is the wavelength separation between resonances) and large extinction ratio (resonance depth) are required, which are also the main challenges today. Therefore, for modern broadband applications, highly dynamic performance for filters are required and for this various methods are used. One common method is using microelectro mechanical system. In this system, the waveguide is completely uncoupled at zero bias, when a voltage is applied on the electrodes, the waveguides are bent towards the microdisk, thus increasing the coupling strength between electrodes. Based on this method, filters with adjustable attenuation have been achieved, and similarly a filter with tuneable bandwidth from $2.8 \mathrm{GHz}$ to $78.4 \mathrm{GHz}$ has also been reported later.

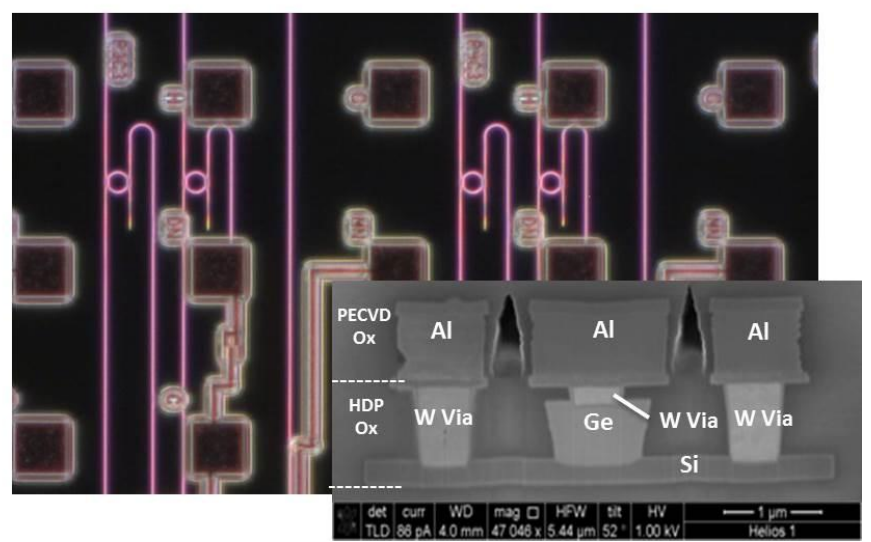

Fig. 2. shows the ring resonator filters and Ge detector grown on Si waveguide. many leading-edge silicon photonics devices widely used in communications, sensing, and computing is demonstrated using this silicon photonics platform.

\section{MODULATORS}

A modulator is a device which changes the fundamental characteristics of a light beam with respect to an information signal. Scientists from Intel Corporation stated that silicon-based optical modulator can operate at $10 \mathrm{~Gb} / \mathrm{s}$. The structure of n-type crystalline silicon consists of an upper "rib" of p-type silicon created by epitaxial lateral overgrowth(ELO) connected to it. These n-type and p-type regions are unconnected by a thin insulating layer of oxygen. The refractive index distribution in the device gets changed when we apply a positive voltage across p-type silicon as charge carriers get accumulated near oxide interface. Generally, this device achieves modulation depth of 3.8$\mathrm{dB}$ at voltage of $1.4 \mathrm{~V}$ and has an electrooptic modulation metric of $3.3 \mathrm{Vcm}$. certain factors should be considered while evaluating the performance of a modulator. The first one is modulation speed, which is the movement of data at a certain rate. The second one is bandwidth which represents the frequency at which the modulation is reduced to $50 \%$ of the maximum value. The third one is insertion loss which informs about the loss in signal power resulting from the insertion of a device in any transmission line or optical fibre. The fourth is area efficiency (size or footprint), and power consumption. the last is modulation depth, also known as extinction ratio that means ration between the optical intensity at maximum transmission and minimum transmission. A modulation depth greater than $7 \mathrm{~dB}$ is always preferred for interconnection purpose. 


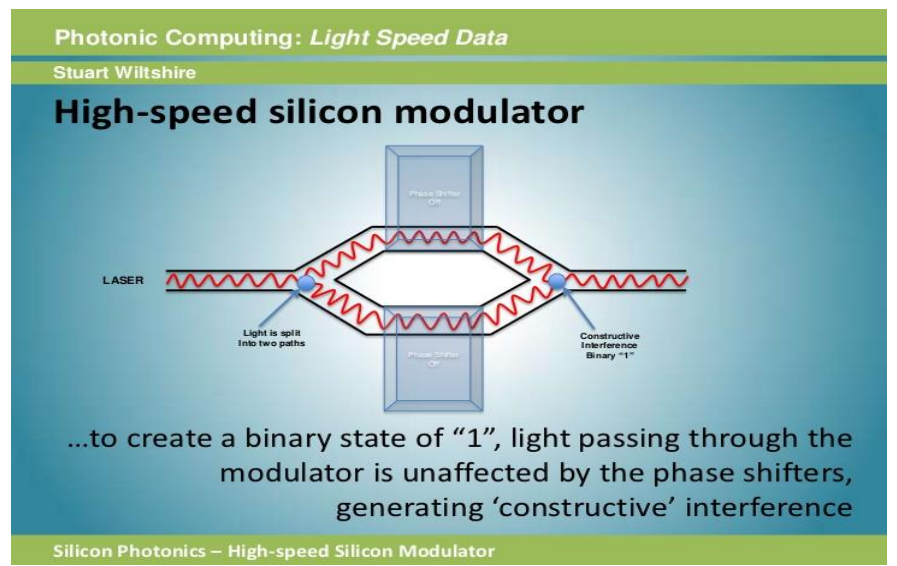

Fig. 3. shows a picture of silicon modulator in which a laser beam is splitted in 2 parts and create

A compact device was discovered using a ring resonator (10 $\mu \mathrm{m}$ diameter) for considering all these factors by Lipson et al. at Cornell University. The highly scaled devices use waveguides of $450 \mathrm{~nm}$ in width and $250 \mathrm{~nm}$ in height. Free carriers can be introduced optically into this medium via two-photon absorption (TPA) with help of ultra-short pulses as well as electrically via carrier injection. This enhancement in power of the resonant structure decreases the power requirement for switching. Modulation depths up to $15 \mathrm{~dB}$ were measured with less than $0.3-\mathrm{V}$ bias voltage change in the electrical version. An alternative approach for optical modulation is to employ integrate organic materials with silicon devices. LiNbO3 is one them which exhibit strong second-order nonlinear electrooptical effects, such as Pockels effect, which gives them excellent candidates for modulation application. LiNbO3 modulators have been developed for commercial uses as they provide good performance of $20 \mathrm{~dB}$ modulation depth, $20 \mathrm{~Gb} / \mathrm{s}$ bandwidth, and $4 \mathrm{~V}$ switch voltage. Up to date, ultrafast LiNbO3 modulators with modulation speed at $110 \mathrm{GHz}$ have been achieved by using titanium (Ti) diffusing while ultralow driving voltage at $2 \mathrm{~V}$ and bandwidth of $15 \mathrm{GHz}$ are also realized. One problem of LiNbO3 modulators is that they are too expensive, therefore it will be very competitive in future whether which dominate in the field of optical modulators.

\section{DETECTORS}

Photodetectors are the devices which convert the received optical signals into electrical signals for further processing in electrooptical integrated circuits. These consist of $p-n$ or $p-i-n$ structures of photodiodes which usually works under a reverse bias voltage in a close loop. While photons are absorbed by $\mathrm{p}-\mathrm{n}$ junction, by applying the built-in electric field across it, hole-electron pairs are formed i.e. holes in $\mathrm{n}$ area and electrons in $\mathrm{p}$ area. By observing the produced photocurrent produced in this process, the corresponding optical signals can be detected.

The performance of a photodetector can be increased by considering these three factors, namely, dark current, bandwidth and responsivity. Dark current is the constant response current exhibited by the detectors without any input light signals. This creates noise at carriers as this current flows with signal currents created from detectors. This noise ultimately increases the power consumption and reduces the sensitivity. The converting speed of a detector is determined by its bandwidth, which can be controlled by changing the transition time for photocarriers to travel from junction to contacts. Responsivity refers to the ratio of collected photocurrent to the optical power incident on detectors for evaluating the receiving efficiency of a photodetector.

Since Silicon shows transparency behaviour in the $1300-1550 \mathrm{~nm}$ operating wavelength range, it is not a suitable material for fibre-optic communication application. Whereas, it can work excellently for applications like as X-ray or gamma-ray detectors for biomedical purposes and space imaging at wavelength below $1000 \mathrm{~nm}$, where band-to-band absorption happens. To remove this transparency problem, there are three main solutions explained one by one below. These methods are implemented using Schottky structure, III-V heterogeneous detectors and Ge detectors constructed on silicon substrates.

The first method is using Schottky structure, here employ Schottky metal-silicon (MS) is employed instead of traditional p-n junction. The reason we use them is that they are having extremely high switching speed and simple fabrication process, which is compatible with standard CMOS technology. There is also one drawback that its quantum efficiency is very low due to the leakage photon flux within the metallic layer.

The second method is using the detectors based on III-V material. We use these because of their wider absorption bandwidth, with relative low dark currents as compared to that of silicon. Current In Ga As PIN photodetectors can operate at a responsivity of more than $1 \mathrm{~A} / \mathrm{W}$ and very low dark current of around several picoamperes. Now a days, a smart use of two-photon absorption behaviour in III-V materials can also be applied for gaining low-speed detection of below-bandgap radiation, and such devices can be used as auto correlators in lasers for pulse generation. 
The third method is using Ge detectors built on silicon substrates. Ge element has smaller bandgap due to which it has stronger absorption at required wavelengths creating layers of $\mathrm{GeSi}$ as well as $\mathrm{SiGeC}$ alloys.

The lattice constant of $\mathrm{Ge}$ is $4 \%$ larger than that of $\mathrm{Si}$. The strain resulting from lattice mismatch modifies the band structure and causes dislocation defects that increase the leakage current of the p-i-n photodetector. Interestingly, by scaling the device to smaller dimensions, a higher dark current can be tolerated.

Another great example of detectors is Avalanche photodiodes (APDs). The main advantage of using these detectors is that they exhibit $5 \sim 10 \mathrm{~dB}$ better sensitivity than p-i-n type detectors due to internal multiplication property present in APDs. They are mainly used in fields where applications like 3D imaging and single-photon detection are needed. The structural diagram of detector is shown in Fig. 4. below:

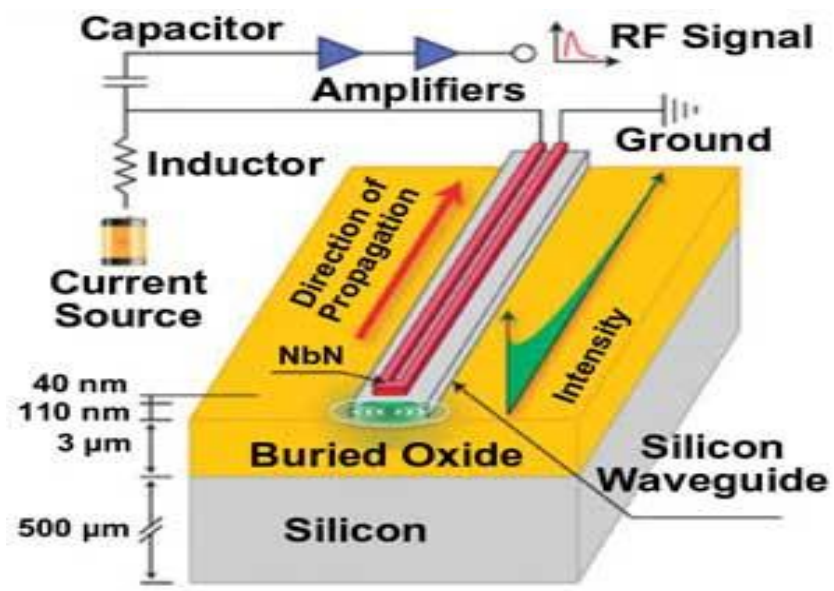

Fig. 4. The single-photon detector is characterized by five convincing factors: $91 \%$ detection efficiency; negligible dark counting rates; high timing resolution; direct integration on chip and counting rates on a gigahertz scale.

\section{AMPLIFIERS AND LASERS}

The creation of silicon optical amplifiers and lasers has often been considered as the progress in silicon photonics by mature silicon processing technology with low cost and high functionality. Optical amplifiers are key components in realizing high levels of photonic integration as they are remunerated for all optical losses.

Silicon has unusual band structure in which, conduction and valence bands do not occur at the same value of crystal momentum. The de-excitation of an electron needs to be negotiated by emitting or absorbing a phonon to conserve momentum because visible phonons have negligible momentum. These radiative recombination events do not occur frequently, as lifetime is very large i.e. about $1 \mathrm{~s}$. The experimentally calculated lifetime of silicon is present in range of millisecond to microsecond, depending on the impurity or defect concentration. This concept leads us to situation where the desired radiative processes are insignificant compared to undesired non radiative recombination. There are numerous approaches have been explored this problem, most of them belong to the four categories given below:

\section{A. $\quad$ EPITAXIAL III-V-BASEd LASERS ON SILICON}

Another main branch to obtain light sources in silicon networks is to implement idea of epitaxial III-V-based materials, such as GaAs and InP, on Si substrates. The main obstacle for this method is that compared with $\mathrm{Si}$, GaAs and InP have lattice mismatches of $4.1 \%$ and $8.1 \%$ and thermal expansion coefficient mismatches of $120.4 \%$ and $76.9 \%$, respectively. Still plenty of approaches have been accessed to integrate III-V layers on SOI substrates.

\section{B. Light EMISSION IN BULK SILICON}

We know that, there are slow optical transition rates present in this system but there are also two major nonradiative processes in $\mathrm{Si}$, which also limit the emission efficiency. The first process is the free-carrier absorption (FCA), in which free carriers in conduction band will absorb photons and jump to higher energy levels. The second one is Auger recombination, in which an electron or hole is excited to a higher energy level by absorbing the energy released from an electron-hold recombination.

\section{QuANTUM CONFINEMENT AND RARE-EARTH IONS DOPING}

Since the lifetime of radiative combination is much larger than that of non-radiative combination, they acquire sufficient time which is to be needed for excited carriers to obtain suitable phonon before they even travel away from some defects in silicon and consecutively loss its pumped energy through heat emissions. One easy method to overcome this situation is through application of quantum confinement effect. It is observed that carriers get localized 
and their momentums also become uncertain, when the size of silicon crystal is reduced to nanometric level. This will in turn improve the probability of radiative recombination.

\section{STIMUlated RAMAN ScAtTERING}

The Raman scattering refers to the inelastic scattering of a photon. The system gets excited to a higher state when it absorbs incident light of wavelength $\omega p$. Now the energy is quickly released by scattering a photon, which is known as Rayleigh scattering. Still then, there is still very small fraction of incident photons (approximately one in ten million) that experiences an excitation due to the thermal vibration of the lattice at frequency $\omega v(15.6 \mathrm{THz}$ in $\mathrm{Si})$.

Another successful approach for epitaxial lasers is using Ge-on-Si structure. Ge has a direct bandgap of $0.8 \mathrm{eV}$, corresponding to the required telecommunication wavelength around $1550 \mathrm{~nm}$, and a sufficiently higher compatibility with silicon CMOS processes, which makes it possible to achieve monolithic integrated devices on silicon, including modulators, photodetectors, and now lasers. Ge has ability to become a potential candidate for lasers in OEIC.

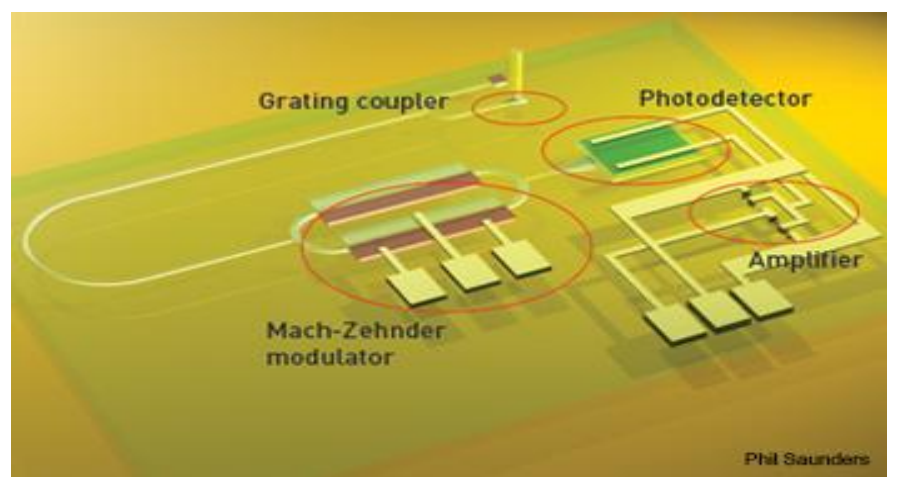

Fig. 5. shows the links between amplifier and other devices.

\section{APPLICATIONS}

Many devices for commercial purposes are being explored by research and development committee and they are still working on that topics. Some of successful applications are -

\section{A. Optical communication}

Silicon photonics is widely used in optical interconnections for on-chip and chip to chip communications. Optical communication is any type of communication in which light is used to carry the signal to the remote end, instead of electrical current. Variations of electron and hole densities change the real and the imaginary part of the refractive index of silicon as described by the empirical equations of Soref and Bennett. A light signal, a transmitter/receiver, modulator/demodulator, and a transparent channel are the building blocks of the optical communications system. Nonresonant modulators, such as Mach-Zehnder interferometers, have typical dimensions in the millimetre range and are usually used in Telecom or Datacom applications. Optical communication systems consist of three major the following components.

1) Transmitter: Converts and transmits an electronic signal into a light signal. for example, light-emitting diodes (LEDs) and laser diodes.

2) Receivers: Typically consist of a photo-detector, which converts light into electricity using the photoelectric effect.

3) Optical Fiber(Repeater): It has core, cladding and a buffer present it it, through which the cladding controls the light along core by simply using total internal reflection(TIR).

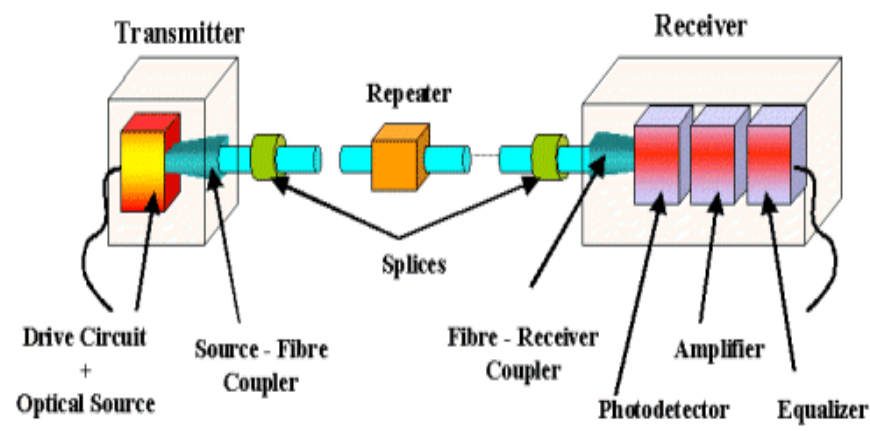

Fig. 6. Optical Communication 
The major part of silicon photonic communications have been limited to telecom and datacom applications only till now, whereas it is expected to reach several kilometres or several meters respectively.

Silicon photonics, however, is expected to play a crucial role in computercom as well in future, where optical links have a reach in the centimeter to meter range. Intel Corporation launched their advanced circuits and technology integration project, in which optical connection is used to replace electrical interconnect for chip-to-chip communication, and the bandwidth is expected to be $200 \mathrm{~Gb} / \mathrm{s}$ to $1 \mathrm{~Tb} / \mathrm{s}$. Three-dimensional integration for nanophotonics has also been proposed as an excellent way to improve the performance of silicon photonics these years.

\section{B. Switches within optical routers}

Another important application of silicon photonics is in signal routers used for optical interconnections. An optical switch is a switch which enables signals of integrated optical circuits switch from one circuit to another circuit. An optical switch works on mechanical mechanisms such as physically shifting an optical fiber to drive one or more alternative fibers one by one and can be performed by magneto-optic effects and electro-optic effects also. Slow optical switches, such as those using moving fibers, may be used for alternate routing of an optical switch transmission path, whereas Fast optical switches, such as those using magneto-optic or electro-optic effects, may be used for performing logic operations. The optical routers use optical switching techniques in order to work properly. These routers are able to give IP at a speed of light. The role of any router is to deliver the intended packets to its required destination. Optical routers have multiple input and output ports embedded in them, equipped with large number of linecards. There is a polarization controller installed between the plurality of transmitters and plurality of input ports. A fourth generation router is shown below in Fig. 7.

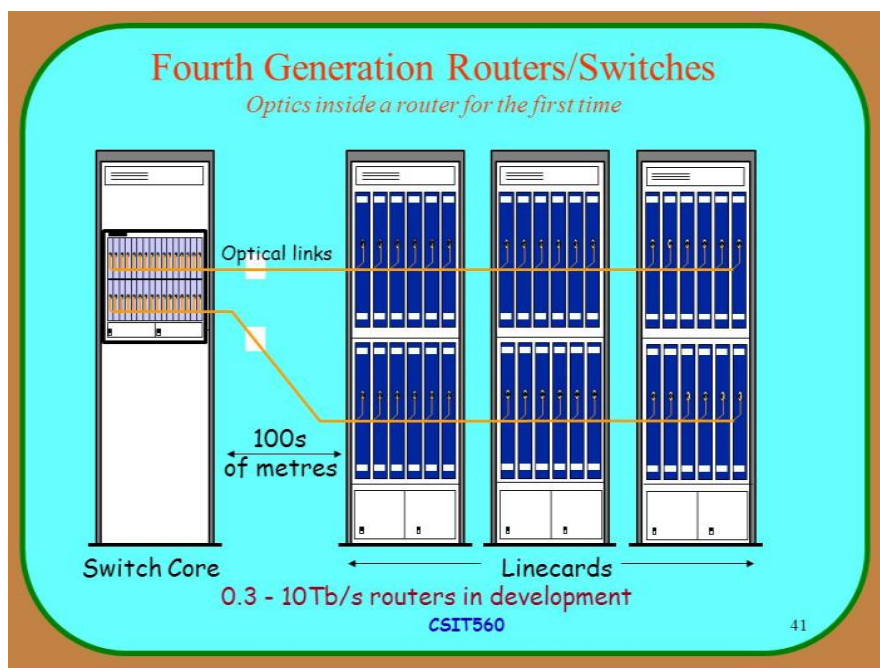

Fig. 7. fourth generation router

The high speed optical routers help all ISPs to cater the needs of their customers as well as enabling them with many value added services. Some of the optical routers work so well that they can provide a speed of up to $256 \mathrm{Gbps}$.

C. 3DICs/integrated optoelectronic chips

Silicon photonics is widely used in Emerging products like 3DICs/integrated optoelectronic chips. Here two or more layers of active electronic components are integrated horizontally and vertically into a single circuit. In 3D design structure of $\mathrm{Si}$, it is completely divided in number of layers of oxide and metal, to form transistors. Thus, it reduces chip area and power dissipation by increase in transistor package density.

\section{Medical applications}

It is used in Medical applications like DNA, glucose, molecular and cellular analysis sensors. Silicon nanophotonics has the transformative potential to produce low-cost, highly integrated medical biosensors for healthcare diagnostic applications and point-of-care (POC) clinics. By leveraging existing CMOS fabrication processes and their economies of scale, silicon photonic sensors offer significant advantages over traditional bio sensing platforms, such as thousands of sensors assembled on a single millimetre-scale chip.

\section{E. Military applications}

It is applied in Military/aerospace/scientific sensor, control and interconnect applications. Many technological opportunities have been made possible through advancement in optics and photonics since the National Research 
Council's (NRC's) publication in 1998 on Harnessing Light: Optical Science and Engineering for the 21st Century. Laser weapons are poised to cause a revolution in military affairs, and integrated optoelectronics is on the verge of replacing many traditional integrated circuit functions. There has been remarkable progress in both laser power and available wavelengths for applications present in national defense training programs. There are several advancements found in system of laser technology. One example is present in area of optical aperture synthesis, which rapidly expanded from the laboratory to flight system demonstrations since Harnessing Light ever appeared within several years.

\section{F. Optical sensors}

Optical sensors, for example, analyze organic and inorganic substances from gaseous, liquid and solid samples. Optical instruments are used in microscopy, spectroscopy and medical imaging. Several examples for different sensing purposes, such as biomedicine, gas, evanescent or optical field, have been demonstrated successfully. One of the example shows an evanescent field sensor for DNA molecules sensing in Silicon on Insulator. The electric field of waveguide mode has an evanescent tail that extends outside of the core layer. This tail interacts with the molecules above and changes the effective refractive index of the waveguide below, and the change in index depends on the size and density of the absorbed molecules. By the way, silicon photonics is also employed and used for other applications, such as imaging purposes, solar cells and lighting devices. An example of optical sensor is shown below in Fig. 8.

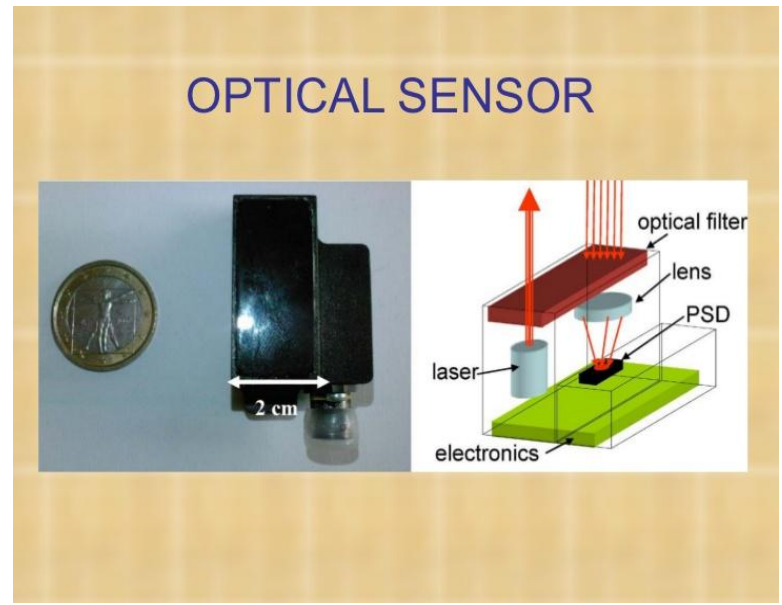

Fig. 8. Optical Sensor

Silicon photonic systems are applied where Optical signal processing with high performance active elements including VOAs and phase, amplitude and frequency modulators for advanced modulation formats are needed. Functional passive optical elements including AWGs, optical filters, couplers and splitters also works on this technology.

\section{FUTURE OF SILICON PHOTONICS}

In future, early stage of silicon photonics may struggle finding low-volume opportunities in market because silicon photonics is attractive method and most affordable due to its Si-CMOS infrastructure. This will not be a bad news, but still as we know new technologies often begin in low volume quantity only. It will be crucial to decide the cost, performance, and size required for integrating photonics onto the same chip as electronics in various applications. Research also shows that for commercial purposes using InP is also efficient instead of using Si. Designs of InP are equivalent to silicon-platform alternatives at all production volumes. They are even much cheaper than silicon productions. The outcomes of this method conclude that there will be significant values in investment of InP platforms having monolithic integration technologies and standardizing production. Optical data transmission increases the data rate transmission and eliminates the complication of electromagnetic interference. The speed of data transmission is so much that we can perform videoconferencing in high resolution with our mates or family members fluently. Advancement in the field of silicon nanophotonic technologies will certainly lead human era to advanced objectives in future computing systems.

\section{ADVANTAGES}

- Silicon photonics is widely used in variety of devices like modulators, integrated photodetectors, optical couplers, wavelength splitters and combiners.

- High index contrast for compact optical circuitry. 
- It is distance independent.

- Higher bandwidth is obtained.

- Through this technology, we can assemble Nano photonic components like ring resonators and filters.

- High speed modulation is attained here (about more than $50 \mathrm{Gbps}$ ).

- Operates in important Datacom and Telecom wavelengths (O- and C-Bands at 1310 and $1550 \mathrm{~nm})$

- Integrated photodetectors via monolithic germanium integration.

- Widely used in multi-pocket system.

- Low-energy consumption.

- Dense Wavelength Division Multiplexing (DWDM) is achieved.

- There are no pin limits, so no scalability.

- Monolithic integration using commercial semiconductor chip foundries enables repeatable, low cost products at scalable volumes.

\section{DISADVANTAGES}

- Silicon has poor optical emission efficiency due to its indirect band-gap by which it suffers from light sources.

- For these reasons, R\&D focuses on the development of practical silicon light sources. Lasers are preferred as a light source but it requires high pumping mechanism.

- Precise alignment is required for Optical connections.

- Band-gap of silicon is larger than desirable that makes it impossible to detect light in the telecom spectral range 1.5 and $1.3 \mu \mathrm{m}$.

- Power dissipation of components is increasing day by day across the chips as these components are getting integrated.

- Integrating photonic components on the same chip requires additional fabrication steps.

\section{CONCLUSION}

In conclusion, I have demonstrated various properties of silicon photonics and its uses in detection, generation and processing of light. This research paper focuses the theoretical discussion of basic principle of silicon photonics to provide low power consumption and high operating speed in the field of data transfer.

\section{REFERENCES}

[1] C. Z. Zhao, E. K. Liu, G. Z. Li, N. Li, and L. Guo, "Silicon raised strip waveguides based on silicon and silicon dioxide thermal bonding," IEEE Photonics Technology Letters, vol. 9, no. 4, pp. 473-474, 1997.

[2] R. A. Soref and J. P. Lorenzo, "All-silicon active and passive guided-wave components for $\lambda=1.3$ and $1.6 \mu \mathrm{m}$," IEEE Journal of Quantum Electronics, vol. 22, no. 6, pp. 873-879, 1986.

[3] B. Jalali, S. Yegnanarayanan, T. Yoon, T. Yoshimoto, I. Rendina, and F. Coppinger, “Advances in silicon-on-insulator optoelectronics," IEEE Journal on Selected Topics in Quantum Electronics, vol. 4, no. 6, pp. 938-947, 1998.

[4] nt, and future of silicon photonicse", IEEE J. Sel. Top. Quantum Electron, 12, (6), pp. 1678-1687, 2006.

[5] Ali Okey, Thesis, SI-GE Photodetection Technologies for integrated optoelectronics, partialfulfilment of doctraldiss, Stanford University, 2007.

[6] Ivan S. Maksymov,"Optical switching and logic gates with hybrid plasmonic-photonic crystal nanobeam cavities "Physics Letters A 375 ,2011 918-921.

[7] T. K. Liang and H. K. Tsang, "Role of free carriers from two-photon absorption in Raman amplification in silicon-on-insulator waveguides," Appl. Phys. Lett., vol. 84, no. 15, pp. 2745-2747, Apr. 2004.

[8] R. Claps, V. Raghunathan, D. Dimitropoulos, and B. Jalali, "Influence of nonlinear absorption on Raman amplification in silicon waveguides," Opt. Express, vol. 12, no. 12, pp. 2774-2780, Jun. 2004

[9] M. A. Mendicino, "Comparison of properties of available SOI materials," in Properties of Crystalline Silicon, R. Hull, Ed. London, U.K.: Inst. Eng. Technol., 1998, ch. 18.1, pp. 992-1001.

[10] J. L. Freeouf and S. T. Liu, "Minority carrier lifetime results for SOI wafers," in Proc. IEEE Int. SOI Conf., Oct. 1995, pp. 74-75.

[11] D.Dimitropoulos, R.Jhaveri,R.Claps,J.C.S.Woo,andB.Jalali,"Lifetime of photogenerated carriers in silicon-on-insulator rib waveguides," Appl. Phys. Lett., vol. 86, no. 7, pp. 071115(1)-071115(3), Feb. 2005.

[12] R. Espinola, J. Dadap, R. Osgood, S. J. McNab, and Y. A. Vlasov, "Raman amplification in ultrasmall silicon-on-insulator wire waveguides," Opt. Express, vol. 12, no. 16, pp. 3713-3718, Aug. 2004.

[13] "Low-loss, single-mode, optical phase modulator in SIMOX material,” J. Lightw. Technol., vol. 12, no. 8, pp. 1394-1400, Aug. 1994.

[14] R. A. Soref and B. R. Bennett, "Kramers-Kronig analysis of E-O switching in silicon," in Proc. SPIE Integr. Opt. Circuit Eng., 1986, vol. 704, pp. 32-37.

[15] C. K. Tang and G. T. Reed, “Highly efficient optical phase modulator in SOIwaveguides,"Electron.Lett.,vol.31,no.6,pp.451-452,Mar.1995.

[16] G. T. Reed and C. E. Png, "Silicon optical modulators," Mater. Today, vol. 8, no. 1, pp. 40-50, Jan. 2005

[17] Y. Liu, C. W. Chow, W. Y. Cheung, and H. K. Tsang, "In-line channel power monitor based on helium ion implantation in siliconon-insulator waveguides," IEEE Photon. Technol. Lett., vol. 18, no. 17, pp. 1882-1884, Sep. 2006

[18] L. Pavesi and G. Guillot, Optical Interconnects: The Silicon Approach. Springer Series in Optical Sciences. New York: Springer-Verlag, 2006.

[19] R. A. Soref and B. R. Bennett, "Electrooptical effects in silicon," IEEE J. Quant. Electronics, vol. QE-23, no. 1, pp. 123-129, Jan. 1987. 